\title{
Author Index to Volume 27 - 2000
}

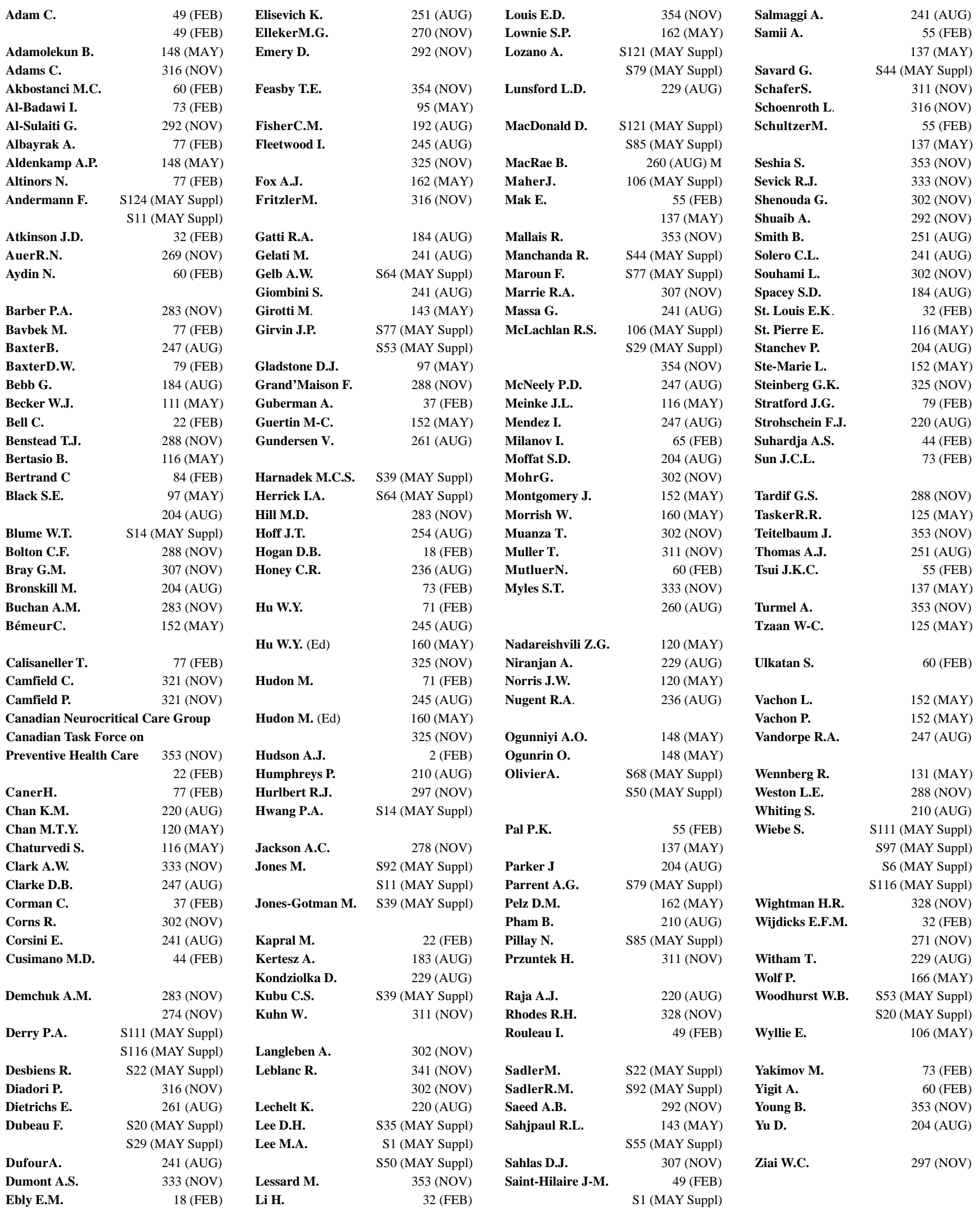




\section{Subject Index to Volume 27 - 2000}

ABSTRACTS

Abstracts: 35th Meeting of the Canadian Congress of Neurological Sciences

ADHESION MOLECULE

High-Dose Methylprednisolone Reduces Cytokine-Induced Adhesion Molecules on Human Brain Endothelium 241 (AUG)

AGING

Predicting Who Will Develop Dementia in a Cohort of Canadian Seniors

18 (FEB)

\section{ALZHEIMER DISEASE}

Editorial: Predicting Dementia in the Elderly: a Physician-friendly Formula

Predicting Who Will Develop Dementia in a Cohort of Canadian Seniors

Callosal Atrophy Correlates with Temporal Lobe Volume and Mental Status in Alzheimer's Disease 204 (AUG)

AMYOTROPHIC LATERALSCLEROSIS

Neuroimaging Highlight: Amyotrophic Lateral Sclerosis 160 (MAY)

\section{ANEURYSMS}

Endovascular Therapy of a Large Vertebral Artery Aneurysm using Stent and Coils162 (MAY)

Endovascular Treatment of a "Blister-like"

Aneurysm of the Internal Carotid Artery 247 (AUG)

ANTICONVULSANT

Generic Substitution for Brand Name Antiepileptic Drugs: A Survey

37 (FEB)

ANTIEPILEPTIC DRUGS

Generic Substitution for Brand Name Antiepileptic Drugs: A Survey

Letter to the Editor: Management of Parkinson's Disease: A Review of Current and New Therapies

Management of Antiepileptic Drugs following Epilepsy Surgery: A Review S106 (MAY Suppl)

Seizure Control as an Indicator of Therapeutic Usefulness S97 (MAY Suppl)

Letter to the Editor:

Neuroprotection and Parkinsonism 261 (AUG)

\section{ARTERIALDISSECTION}

Vertebral Artery Dissection: Warning Symptoms, Clinical Features and Prognosis in 26 Patients

$292($ NOV)

ARTERIOVENOUS MALFORMATIONS

Neuroimaging Highlight: Cerebral Cavernous Malformations 245 (AUG) Neuroimaging Highlight: Moyamoya Disease

325 (NOV)

ASTROCYTOMAS

The Role of Stereotactic Cyst Aspiration for Glial and Metastatic Brain Tumors 229 (AUG)

High Dose Tamoxifen and Radiotherapy in Patients with Glioblastoma Multiforme: A Phase IB Study

$302(\mathrm{NOV})$

ATAXIA

The Molecular Basis and Clinical Management of Ataxia Telangiectasia 184 (AUG)

BRAIN DEATH

Letter to the Editor: Apnea test in the diagnosis of brain death

353 (NOV)

Letter to the Editor: Response to Lessard et al 353 (NOV)

\section{BRAIN STEM}

Nucleus of the Tractus Solitarius Metastasis: Relationship to Respiratory Arrest? 328 (NOV)

BRAIN TUMOURS

The Role of Stereotactic Cyst Aspiration for Glial and Metastatic Brain Tumorss 229 (AUG) High Dose Tamoxifen and Radiotherapy in Patients with Glioblastoma

Multiforme: A Phase IB Study 302 (NOV)

Nucleus of the Tractus Solitarius Metastasis: Relationship to Respiratory Arrest? 328 (NOV)

Familial Adenomatous Polyposis and

Benign Intracranial Tumors:

A New Variant of Gardner's Syndrome 341 (NOV)

CARDIOLOGY

Use of Ambulatory Electrocardiography for the Detection of Paroxysmal Atrial Fibrillation in Patients with Stroke

22 (FEB)

\section{CAVERNOUS ANGIOMA}

Neuroimaging Highlight:

Cerebral Cavernous Malformations 245 (AUG)

CEREBELLUM

Predictors of Poor Outcome in Patients

with a Spontaneous Cerebellar Hematoma32 (FEB)

CEREBRAL PALSY

Hemiparetic Cerebral Palsy: Clinical Pattern and Imaging in Prediction of Outcome 210 (AUG)

CEREBROVASCULAR DISEASE

Use of Ambulatory Electrocardiography for the Detection of Paroxysmal Atrial Fibrillation in Patients with Stroke

Diagnostic Strategies in Young Patients with Ischemic Stroke in Canada

120 (MAY)

COGNITIVE IMPAIRMENT

Cognitive Function in Nigerians with Newly Diagnosed Epilepsy

148 (MAY)

COMA

Letter to the Editor: Apnea test in the diagnosis of brain death

353 (NOV)

Letter to the Editor:

Response to Lessard et al

$353(\mathrm{NOV})$

COMPUTED TOMOGRAPHY

Predictors of Poor Outcome in Patients with a Spontaneous Cerebellar Hematoma32 (FEB)

\section{CYTOKINE}

High-Dose Methylprednisolone Reduces Cytokine-Induced Adhesion Molecules on Human Brain Endothelium 241 (AUG)

DEGENERATIVE DISEASES - DEMENTIA

Editorial: Alien Hand, Free Will and Arnold Pick

$183(\mathrm{AUG})$

Alien Hand Phenomena: A Review with The Addition of Six Personal Cases

Callosal Atrophy Correlates

with Temporal Lobe Volume and Mental

Status in Alzheimer's Diseas

204 (AUG)

DEMENTIA

Editorial: Predicting Dementia in the Elderly: a Physician-friendly Formula 17 (FEB) Predicting Who Will Develop Dementia in a Cohort of Canadian Seniors

18 (FEB)

Editorial: Alien Hand, Free Will and Arnold Pick

Alien Hand Phenomena: A Review with The Addition of Six Personal Cases

Callosal Atrophy Correlates with Tempora Lobe Volume and Mental Status in Alzheimer's Disease

\section{DISC HERNIATION}

Ogilvie's Syndrome as a Rare Complication of Lumbar Disc Surgery

77 (FEB)

DYSTONIA

Post-traumatic Cervical Dystonia: A Distinct Entity?

Head Tremor in Cervical Dystonia

137 (MAY)

\section{ECONOMICS}

Are the Triptans for Migraine Therapy Worth the Cost?

111 (MAY)
EDITORIAL

Editorial: Endarterectomy for Asymptomatic Carotid Stenosis in the Real World 95 (MAY)

ELECTROCARDIGRAPHIC

Use of Ambulatory Electrocardiography for the Detection of Paroxysmal Atrial

Fibrillation in Patients with Stroke

ELECTROENCEPHALOGRAPHY

Postictal Aphasia and Paresis:

A Clinical and Intracerebral EEG Study 49 (FEB)

Electroclinical Analysis of Postictal

Noserubbing

131 (MAY)

Scalp EEG in Temporal Lobe Epilepsy Surgery

S22 (MAY Suppl)

Invasive Electrographic Recording Techniques

in Temporal Lobe Epilepsy S29 (MAY Suppl)

Investigation

S50 (MAY Suppl)

How Often Does Routine Pediatric EEG have an Important Unexpected Result? 321 (NOV)

\section{ELECTROMYOGRAPHY}

Difference of Disability Between Electrophysiologic Subgroups of Essential Tremor 60 (FEB)

Clinical and Electromyographic Examinations of Patients with Essential Tremor 65 (FEB)

Specialty Societies and Practice Standard Setting - Whose Job is it Anyway? 270 (NOV)

Minimum Standards for Electromyography in Canada: A Statement of the Canadian Society of Clinical Neurophysiologists 288 (NOV)

\section{ELECTROPHYSIOLOGY}

Specialty Societies and Practice Standard Setting - Whose Job is it Anyway?

Minimum Standards for Electromyography in Canada: A Statement of the Canadian Society of Clinical Neurophysiologists 288 (NOV)

EMBOLI

Use of Ambulatory Electrocardiography for the Detection of Paroxysmal Atrial Fibrillation in Patients with Stroke

\section{ENCEPHALITIS}

Rabies 278 (NOV)

ENDARTERECTOMY

Attitudes of Canadian and U S Neurologists Regarding Carotid Endartherectomy for Asymptomatic Stenosis 116 (MAY) Editorial: Endarterectomy for Asymptomatic Carotid Stenosis in the Real World 95 (MAY)

ENGLISHSTYLE

The Alphabetization of Journal Articles 354 (NOV) EPIDEMIOLOGY

Epidemiology of Temporal Lobe Epilepsy

S6 (MAY Suppl)

EPILEPSY

Generic Substitution for Brand Name Antiepileptic Drugs: A Survey

37 (FEB)

Postictal Aphasia and Paresis: A Clinical and Intracerebral EEG Study 49 (FEB) Surgical Treatment of Epilepsy in Pediatric Patients

Management of Antiepileptic Drugs following Epilepsy Surgery: A Review

Electroclinical Analysis of Postictal Noserubbing

Cognitive Function in Nigerians with Newly Diagnosed Epilepsy

Epilepsy in Comtemporary Fiction: Fates of Patients 06 (MAY Suppl)

131 (MAY)

ocalizing and Lateralizing Value of Epileptic Symptoms in Temporal Lobe Epilepsy

148 (MAY) Lobe Epilepsy Surgery: Definition of Candidacy 
Measuring Quality of Life in

Epilepsy Surgery Patients S111 (MAY Suppl)

Psychological Adjustment to Success, to Failure following Epilepsy Surgery S116 (MAY Suppl)

Summary

Pediatric Candidates of Temporal Lobe Epilepsy Surgery S124 (MAY Suppl)

Identification of Candidates

Scalp EEG in Temporal Lobe Epilepsy Surgery

S14 (MAY Suppl) S20 (MAY Suppl)

nvasive Electrograph

S22 (MAY Suppl)

in Temporal Lobe Epilepsy S29 (MAY Suppl)

MRI Techniques: Bilateral Findings and

"Normal Findings" S35 (MAY Suppl)

Neuropsychological Assessment for Temporal Lobe Epilepsy Surgery S39 (MAY Suppl)

Psychiatric Assessment of Candidates for Epilepsy Surgery S44 (MAY Suppl)

Investigation S50 (MAY Suppl)

Communication and Mental Preparation Issues for the Epilepsy Surgery Patient S53 (MAY Suppl)

Awake Craniotomy: Controversies, Indications and Techniques in the Surgical Treatment of Temporal Lobe Epilepsy S55 (MAY Suppl)

Epidemiology of Temporal Lobe Epilepsy

Anaesthesia for Temporal Lobe Epilepsy Surgery

S6 (MAY Suppl) Amygdalohippocampectomy in Temporal Lobe Epilepsy $\quad$ S68 (MAY)

Surgical Pitfalls, their Consequences, Transient Complications S77 (MAY Suppl)

Stereotactic Surgery for Temporal Lobe Epilepsy S79 (MAY Suppl)

Intraoperative Electrocorticography in Temporal Lobe Epilepsy SurgeryS85 (MAY Suppl)

Surgery S92 (MAY Suppl)

Seizure Control as an Indicator of Therapeutic Usefulness S97 (MAY Suppl)

Transient Hemifacial Sensory Loss with Xerophthalmia following Temporal Lobectomy 251 (AUG)

How Often Does Routine Pediatric EEG

have an Important Unexpected Result? 321 (NOV)

EPILEPSYPHARMACOLOGY

Generic Substitution for Brand Name Antiepileptic Drugs: A Survey

37 (FEB)

\section{EPILEPSYSURGERY}

Temporal Lobe Epilepsy Surgery: Definition of Candidacy

Measuring Quality of Life in Epilepsy Surgery Patients S111 (MAY Suppl)

Psychological Adjustment to Success, to Failure following Epilepsy Surgery $\quad$ S116 (MAY Suppl)

Summary

Pediatric Candidates of Temporal Lobe Epilepsy Surgery

S14 (MAY Suppl)

Identification of Candidates

Scalp EEG in Temporal Lobe Epilepsy Surgery S20 (MAY Suppl)

Neuropsychological A

S22 (MAY Suppl)

Psychiatric Assessment of Candidates for Epilepsy Surgery

MAY Suppl)

S44 (MAY Suppl)

Communication and Mental Preparation Issues for the Epilepsy Surgery Patient S53 (MAY Suppl)

Awake Craniotomy: Controversies, Indications and Techniques in the Surgical Treatment of Temporal Lobe Epilepsy S55 (MAY Suppl)

Anaesthesia for Temporal Lobe Epilepsy Surgery S64 (MAY Suppl)

Transcortical Selective Amygdalohippocampectomy in Temporal Lobe Epilepsy $\quad$ S68 (MAY)

Surgical Pitfalls, their Consequences, Transient Complications S77 (MAY Suppl)

Stereotactic Surgery for Temporal Lobe Epilepsy S79 (MAY Suppl)

Intraoperative Electrocorticography in Temporal Lobe Epilepsy SurgeryS85 (MAY Suppl) Surgery S92 (MAY Suppl)

\section{ESSENTIALTREMOR}

Difference of Disability Between Electrophysiologic
Subgroups of Essential Tremor

60 (FEB)

Clinical and Electromyographic Examinations

of Patients with Essential Tremor

65 (FEB)

FMRI

Pain Perception and Response:

Central Nervous System Mechanisms 2 (FEB)

GENETICS - CLINICAL

Neurology and Neurosurgery at the Montreal General Hospital 1960-1980

79 (FEB)

The Molecular Basis and Clinical

Management of Ataxia Telangiectasia 184 (AUG)

GENETICS - MOLECULAR BIOLOGY

The Molecular Basis and Clinical

Management of Ataxia Telangiectasia 184 (AUG)

GLIOMA

High Dose Tamoxifen and Radiotherapy

in Patients with Glioblastoma

Multiforme: A Phase IB Study

302 (NOV)

HEAD TRAUMA

Intracranial Pressure Monitoring in Severe

Traumatic Brain Injury - Results of a

Canadian Survey

143 (MAY)

HEADACHE

Are the Triptans for Migraine Therapy Worth the Cost?

111 (MAY)

\section{HEMORRHAGE}

Predictors of Poor Outcome in Patients with a Spontaneous Cerebellar Hematoma

32 (FEB)

HEMORRHAGE - CEREBRAL

Hemorrhagic Moyamoya Disease during Pregnancy

73 (FEB)

HERNIATION

Hemicraniectomy for Ischemic Stroke: Temerity or Death Cure?

Hemicraniotomy in Massive Hemispheric Stroke: A Stark Perspective on a

Radical Procedure

Hemicraniectomy is a Promising Treatment in Ischemic Stroke

HISTORY

Epilepsy in Comtemporary Fiction: Fates of Patients

Four Early Contributors to

Neurosurgery in North America

$166(\mathrm{MAY})$

Memoriam - Charles W Taylor 1916-1999

254 (AUG)

YDROCEPHALUS

Predictors of Poor Outcome in Patients with a Spontaneous Cerebellar Hematoma

INFARCTION

Use of Ambulatory Electrocardiography for the Detection of Paroxysmal Atria Fibrillation in Patients with Stroke

Hydroxyl Radical Production in the Cortex and Striatum in a Rat Model of

Focal Cerebral Ischemia

152 (MAY)

INFECTIOUS DISEASES

Rabies

Autoantibodies in Childhood Post-Varicella Acute Cerebellar Ataxia

$278(\mathrm{NOV})$

316 (NOV)

\section{INTERFERON}

High-Dose Methylprednisolone Reduces Cytokine-Induced Adhesion Molecules on Human Brain Endothelium 241 (AUG)

INTERVENTIONALNEURORADIOLOGY

Neuroimaging Highlight: Traumatic Carotid - Cavernous Fistula 71 (FEB) Endovascular Treatment of a "Blister-like" Aneurysm of the Internal Carotid Artery 247 (AUG) Neuroimaging Highlight:

Moyamoya Disease

325 (NOV)

MAGNETIC RESONANCE IMAGING

Neuroimaging Highlight:

Amyotrophic Lateral Sclerosis

160 (MAY)

MRI Techniques: Bilateral Findings an

"Normal Findings"

S35 (MAY Suppl)

Callosal Atrophy Correlates

with Temporal Lobe Volume and Mental

Status in Alzheimer's Disease

204 (AUG)

Hemiparetic Cerebral Palsy: Clinical Pattern and Imaging in Prediction of Outcome 210 (AUG)

A Prospective Randomized Comparison of CTand MRI Pre-operative Localization for Pallidotomy

236 (AUG)

\section{MEMORY}

Predicting Who Will Develop Dementia in a Cohort of Canadian Seniors

18 (FEB)

MENINGITIS

Idiopathic Hypertrophic Pachymeningitis: A Report of Two Patients and Review of the Literature

333 (NOV)

METASTATIC TUMOURS

The Role of Stereotactic Cyst Aspiration for Glial and Metastatic Brain Tumorss 229 (AUG)

Nucleus of the Tractus Solitarius Metastasis: Relationship to Respiratory Arrest? 328 (NOV)

MIGRAINE

Are the Triptans for Migraine Therapy Worth the Cost?

111 (MAY)

\section{MOTOR CONTROL}

Age-Related Changes in Muscle Fatigue Resistance in Humans

220 (AUG)

MOTOR NEURON

Age-Related Changes in Muscle Fatigue Resistance in Humans

220 (AUG)

MOTOR PHYSIOLOGY

Age-Related Changes in Muscle Fatigue Resistance in Humans

MOVEMENTDISORDERS

Post-traumatic Cervical Dystonia: A Distinct Entity?

55 (FEB)

Difference of Disability Between Electrophysiologic Subgroups of Essential Tremor

Clinical and Electromyographic Examinations of Patients with Essential Tremor 65 (FEB)

Head Tremor in Cervical Dystonia 137 (MAY)

Letter to the Editor: Issues Relating to Functional Disability in Essential Tremor 354 (NOV)

MOYAMOYA DISEASE

Hemorrhagic Moyamoya Disease during Pregnancy

73 (FEB)

Neuroimaging Highlight: Moyamoya Disease

325 (NOV)

\section{MULTIPLE SCLEROSIS}

High-Dose Methylprednisolone Reduces Cytokine-Induced Adhesion Molecules on Human Brain Endothelium

241 (AUG)

\section{MYASTHENIAGRAVIS}

Familial Autoimmune Myasthenia Gravis: Four Patients Involving Three Generations 307 (NOV)

\section{NEUROANATOMY}

Pain Perception and Response: Central Nervous System Mechanisms

2 (FEB)

\section{NEUROCHEMISTRY}

Hydroxyl Radical Production in the Cortex and Striatum in a Rat Model of Focal Cerebral Ischemia

NEUROLOGICALEXAMINATION

Correlation Between Tapping and Inserting of Pegs in Parkinson's Disease 311 (NOV)

NEUROLOGY- PAEDIATRIC

Autoantibodies in Childhood Post-Varicella Acute Cerebellar Ataxia 316 (NOV)

How Often Does Routine Pediatric EEG Have an Important Unexpected Result? 321 (NOV)

NEUROPATHOLOGY

Canadian Association of Neuropathologists Abstracts of papers and cases presented 
NEURORADIOLOGY

Endovascular Therapy of a Large Vertebral Artery Aneurysm using Stent and Coils 162 (MAY)

Endovascular Treatment of a "Blister-like" Aneurysm of the Internal Carotid Artery 247 (AUG)

\section{NEUROSURGERY}

Craniotomy Revisisted: Techniques for Improved Access and Reconstruction

Intracranial Pressure Monitoring in Severe Traumatic Brain Injury - Results of a Canadian Survey

44 (FEB)

Carly Contributors to

Neurosurgery in North America 254 (AUG)

In Memoriam - Charles W Taylor 1916-1999

Idiopathic Hypertrophic Pachymeningitis: A Report of Two Patients and Review of the Literature

143 (MAY)

$260(\mathrm{AUG})$

$333(\mathrm{NOV})$

\section{NEUROSURGERY - ADULT}

Attitudes of Canadian and U S Neurologists Regarding Carotid Endartherectomy for Asymptomatic Stenosis

116 (MAY)

Percutaneous Radiofrequency Facet Rhizotomy - Experience of 188 Procedures and Reappraisal of its Value

125 (MAY)

Editorial: - Endarterectomy for Asymptomatic Carotid Stenosis in the Real World 95 (MAY)

Transient Hemifacial Sensory

Loss with Xerophthalmia following Temporal Lobectomy

Four Early Contributors to Neurosurgery in North America 254 (AUG)

A Six Year Review of Odontoid Fractures: The

Emerging Role of Surgical Intervention 297 (NOV)

NEUROSURGERY - PAEDIATRIC

Surgical Treatment of Epilepsy in Pediatric Patients

106 (MAY)

\section{NEUROSURGICALPRACTICE}

Craniotomy Revisisted: Techniques for Improved Access and Reconstruction 44 (FEB)

A Six Year Review of Odontoid Fractures: The Emerging Role of Surgical Intervention 297 (NOV)

\section{PAIN}

Pain Perception and Response: Central Nervous System Mechanisms 2 (FEB)

Percutaneous Radiofrequency Facet

Rhizotomy - Experience of 188 Procedures

and Reappraisal of its Value

125 (MAY)

\section{PAIN CONTROL}

Percutaneous Radiofrequency Facet Rhizotomy - Experience of 188 Procedures and Reappraisal of its Value 125 (MAY)

PALLIDOTOMY

A Prospective Randomized Comparison of CTand MRI Pre-operative Localization for Pallidotomy 236 (AUG)

PARKINSON'S DISEASE

Letter to the Editor: Management of Parkinson's Disease: A Review of Current and New Therapies

84 (FEB)

A Prospective Randomized Comparison of CTand MRI Pre-operative Localization for Pallidotomy 236 (AUG)

Letter to the Editor: Neuroprotection and Parkinsonism 261 (AUG) Correlation Between Tapping and Inserting of Pegs in Parkinson's Disease 311 (NOV)

PARKINSONISM

Correlation Between Tapping and Inserting of Pegs in Parkinson's Disease 311 (NOV)

PETSCANNING

Pain Perception and Response: Central Nervous System Mechanisms 2 (FEB)

\section{PHARMACOLOGY}

Generic Substitution for Brand Name Antiepileptic Drugs: A Survey

\section{PRACTICE STANDARDS}

Specialty Societies and Practice Standard
Setting - Whose Job is it Anyway? 270 (NOV)

Minimum Standards for Electromyography in

Canada: A Statement of the Canadian

Society of Clinical Neurophysiologists 288 (NOV)

PREGNANCY

Hemorrhagic Moyamoya Disease

during Pregnancy

73 (FEB)

REHABILITATION

Enhancing Recovery after Stroke with Noradrenergic Pharmacotherapy: A New Frontier?

97 (MAY)

\section{REPLY FROM THE AUTHORS}

Letter to the Editor: Response to Lessard et al

353 (NOV)

Letter to the Editor: Response to The Alphabetization of Journal Articles

$354(\mathrm{NOV})$

\section{SEIZURES}

Postictal Aphasia and Paresis: A Clinical and Intracerebral EEG Study 49 (FEB)

Surgical Treatment of Epilepsy in Pediatric Patients

106 (MAY)

Management of Antiepileptic Drugs following Epilepsy Surgery: A Review 106 (MAY Suppl)

Electroclinical Analysis of Postictal Noserubbing

$131($ MAY)

Cognitive Function in Nigerians with Newly Diagnosed Epilepsy

148 (MAY)

Localizing and Lateralizing Value of Epileptic Symptoms in Temporal Lobe Epilepsy

S1 (MAY Suppl)

Temporal Lobe Epilepsy Surgery: Definition of Candidacy

Measuring Quality of Life in Epilepsy Surgery Patients $\quad$ S111 (MAY Suppl)

Psychological Adjustment to Success, to Failure following Epilepsy Surgery S116 (MAY Suppl)

Summary S124 (MAY Suppl)

Pediatric Candidates of Temporal Lobe Epilepsy Surgery

Identification of Candidates

Scalp EEG in Temporal Lobe Epilepsy Surgery

S14 (MAY Suppl) S20 (MAY Suppl)

S22 (MAY Suppl)

nvasive Electrographic Recording Techniques in Temporal Lobe Epilepsy S29 (MAY Suppl)

MRI Techniques: Bilateral Findings and "Normal Findings" S35 (MAY Suppl)

Neuropsychological Assessment for Temporal Lobe Epilepsy Surgery S39 (MAYSuppl) Psychiatric Assessment of Candidates for Epilepsy Surgery $\quad$ S44 (MAY Suppl)

Investigation S50 (MAY Suppl)

Communication and Mental Preparation Issues for the Epilepsy Surgery Patient S53 (MAY Suppl)

Awake Craniotomy: Controversies, Indications and Techniques in the Surgical Treatment of Temporal Lobe Epilepsy

S55 (MAY Suppl)

Epidemiology of Temporal Lobe Epilepsy

Anaesthesia for Temporal Lobe Epilepsy Surgery 6 (MAY Suppl) S64 (MAY Suppl) Selective Amygdalohippocampectomy in Temporal Lobe Epilepsy S68 (MAY)

Surgical Pitfalls, their Consequences, Transient Complications

S77 (MAY Suppl)

Stereotactic Surgery for Temporal Lobe Epilepsy

S79 (MAY Suppl)

Intraoperative Electrocorticography in Temporal Lobe Epilepsy SurgeryS85 (MAY Suppl)

Surgery

S92 (MAY Suppl)

Seizure Control as an Indicator of Therapeutic Usefulness

S97 (MAY Suppl)

SPINALCORD INJURY

A Six Year Review of Odontoid Fractures: The Emerging Role of Surgical Intervention297 (NOV)

STEREOTACTIC SURGERY

The Role of Stereotactic Cyst Aspiration for Glial and Metastatic Brain Tumors 229 (AUG)

STROKE

Attitudes of Canadian and US Neurologists Regarding Carotid Endartherectomy for

Asymptomatic Stenosis

116 (MAY)

Diagnostic Strategies in Young Patients with Ischemic Stroke in Canada

Hydroxyl Radical Production in the Cortex and Striatum in a Rat Model of

Focal Cerebral Ischemia

120 (MAY)

Focal Cerebral Ischemia

Carotid Stenosis in the Real World

52 (MAY)

nhancing Recovery after Stroke with Noradrenergic Pharmacotherapy: A New Frontier?

95 (MAY)

micraniectomy for Ischemic Stroke: Temerity or Death Cure?

97 (MAY)

emicraniotomy in Massive Hemispheric

Stroke: A Stark Perspective on a Radical Procedure

emicraniectomy is a Promising Treatmen in Ischemic Stroke

Doubts, Fears and Misconceptions What is the Future of Thrombolysis in Acute Stroke 283 (NOV) Vertebral Artery Dissection: Warning Symptoms, Clinical Features and Prognosis in 26 Patients 292 (NOV)

SURGICALCOMPLICATIONS

Ogilvie's Syndrome as a Rare Complication of Lumbar Disc Surgery

77 (FEB)

\section{TEMPORALLOBE}

Localizing and Lateralizing Value of Epileptic Symptoms in Temporal Lobe Epilepsy

S1 (MAY Suppl)

Temporal Lobe Epilepsy Surgery: Definition of Candidacy S11 (MAY Suppl)

Pediatric Candidates of Temporal Lobe

Epilepsy Surgery

S14 (MAY Suppl)

Identification of Candidates

Scalp EEG in Temporal Lobe Epilepsy Surgery S20 (MAY Suppl)

Invasive Electrographic Recording Techniques in Temporal Lobe Epilepsy $\quad$ S29 (MAY Suppl)

MRI Techniques: Bilateral Findings and "Normal Findings"

S35 (MAY Suppl)

Neuropsychological Assessment for Temporal Lobe Epilepsy Surgery S39 (MAY Suppl) Psychiatric Assessment of Candidates for Epilepsy Surgery S44 (MAY Suppl)

Awake Craniotomy: Controversies, Indications and Techniques in the Surgical Treatment of Temporal Lobe Epilepsy S55 (MAY Suppl) Epidemiology of Temporal Lobe Epilepsy

S6 (MAY Suppl)

Transcortical Selective Amygdalohippocampectomy in Temporal Lobe Epilepsy S68 (MAY)

Surgical Pitfalls, their Consequences,

Transient Complications S77 (MAY Suppl) Stereotactic Surgery for Temporal Lobe Epilepsy

S79 (MAY Suppl)

Intraoperative Electrocorticography in Temporal Lobe Epilepsy Surgery $\quad$ S85 (MAY Suppl)

Surgery S92 (MAY Suppl)

Transient Hemifacial Sensory Loss with Xerophthalmia following Temporal Lobectomy 251 (AUG)

\section{THROMBOLYSIS}

Doubts, Fears and Misconceptions - What is the Future of Thrombolysis in Acute Stroke 283 (NOV)

TRAUMA

Post-traumatic Cervical Dystonia:

A Distinct Entity?

Neuroimaging Highlight: 